\title{
The Prognostic Value of Biomarkers on Detecting Non-Small Cell Lung Cancer in a Chinese Elderly Population
}

\author{
Lianghua Guo' \\ Bin Song' \\ Jianhong Xiao' \\ Hui Lin ${ }^{1}$ \\ Junhua Chen' \\ Xianghua $\mathrm{Su}^{2}$
}

'Department of Respiratory Medicine, Mindong Hospital of Fujian Medical University, Fuan City, 355000, People's Republic of China; ${ }^{2}$ Department of Neurosurgery, Mindong Hospital of Fujian Medical University, Fuan City, 355000, People's Republic of China

Correspondence: Xianghua Su Department of Respiratory Medicine, Mindong Hospital of Fujian Medical University, No. 89 Heshan Road, Fuan City, 355000, Fujian Province, People's Republic of China

Email Suxianghua505@I26.com
Background: Survival in non-small cell lung cancer (NSCLC) remains poor. Early detection of NSCLC is of great significance to provide a chance to improve survival.

Aim: We constructed predictive models to evaluate the predictive value of four tumor biomarkers by including serum human epididymis protein 4 (HE4), carcinoembryonic antigen (CEA), squamous cell carcinoma antigen (SCCA), and cytokeratin 19 fragment (CY211) on detecting NSCLC in a Chinese elderly population.

Methods: A total of 363 patients with NSCLC and 433 subjects without cancer (healthy control group) were admitted to the respiratory department in our hospital. We assessed serum levels of these four tumor biomarkers in the two groups and then the predictive value of predictive models was evaluated.

Results: Serum median values of HE4 (143.3 pmol/L), CEA (4.60 ng/mL), SCCA (1.52 ng/ $\mathrm{mL})$, and CY21-1 $(5.36 \mathrm{ng} / \mathrm{mL})$ in patients with NSCLC were significantly higher than the healthy control group, respectively (all $P<0.05$ ). A multivariate logistic regression model showed that HE4 (OR=2.10, 95\% CI=1.22-4.42, $P=0.013)$, CEA $(\mathrm{OR}=2.30,95 \% \mathrm{CI}=1.44$ 4.53, $P=0.004)$, SCCA $(\mathrm{OR}=2.20,95 \% \mathrm{CI}=1.29-4.55, P=0.011)$, and $\mathrm{CY} 21-1 \quad(\mathrm{OR}=2.60$, $95 \% \mathrm{CI}=1.56-6.25, P<0.001)$ were significantly and independently associated with increased risk of NSCLC on admission after confounding factors were corrected. Importantly, the ROC curve suggested HE4 had a good value on predicting NSCLC in the Chinese elderly population. Additionally, the predictive model (CEA+SCCA+CY21-1+HE4) had better idea capability to detecting the existence of NSCLC (AUC $=0.954,95 \% \mathrm{CI}=0.927-0.999$, $P<0.001)$.

Conclusion: Our study showed that several lung cancer-related biomarkers were used to build prediction models, which has good value for early prediction of NSCLC.

Keywords: NSCLC, HE4, CEA, SCCA, CY21-1, Chinese elderly population, predictive value

\section{Introduction}

As one of the common malignant tumors, lung cancer has become the first cause of death in an urban population on a worldwide scale. ${ }^{1}$ Non-small cell lung cancer (NSCLC) accounts for about $80 \%$ of all lung cancer in China. ${ }^{1,2}$ Although the diagnosis and molecular targeted therapy have been developed, about $75 \%$ of these patients with NSCLC are in advanced stage and the survival rate of 5 years has not improved significantly. ${ }^{2}$ Currently, some non-specific tumor biomarkers, including squamous cell carcinoma antigen (SCCA), carcinoembryonic antigen (CEA) and 
cytokeratin 19 fragment (CYFRA 21-1), can improve the sensitivity of diagnosis for NSCLC, but the specificity is still low. ${ }^{3,4}$ Diagnosed NSCLC at an early stage can greatly improve the survival rate of these patients. Therefore, it is necessary to find new serum biomarkers or predictive models that can early and accurately predict the prevalence of NSCLC, providing the treatment opportunity and prolonging survival time for these patients. ${ }^{5}$

Human epididymis protein 4 (HE4), also known as whey-acidic-protein four disulfide core protein-2 (WFDC2), has been considered as a new and promising tumor biomarker, ${ }^{6}$ and the food and drug administration (FDA) has approved serum HE4 as the biomarker for early diagnosing and monitoring of ovarian cancer. ${ }^{7,8}$ HE4 is also a protease inhibitor with protective immunity, involved in sperm maturation in the epididymis epithelium. At present, overexpression of HE4 protein has also been identified in other tumor tissues, like lung cancer, ${ }^{9}$ and high HE4 expression was associated with more serious and shorter survival time. ${ }^{10-12}$ For instance, it has been suggested that HE4 is involved in immune defense and inflammatory response in the oral cavities, nasal and respiratory tract in the development of lung adenocarcinoma. ${ }^{13-15}$ Some published studies also suggested that HE4 expression was associated with the progression of lung cancer. ${ }^{13-19}$ However, investigations of HE4 in NSCLC are still few.

In this study we analyzed serum biomarkers (HE4, CEA, SCCA, and CY21-1) in 363 patients with NSCLC and 433 healthy control subjects, with the aim of searching for new candidate biomarkers or prediction models with the potential to improve the performance of serum biomarkers for discrimination between NSCLC and non-lung cancer. In order to test whether a multiplex biomarker model could further improve the performance of predictive value, a 4-biomarker model (HE4, CEA, SCCA, and CY21-1) was found to be the best model for discrimination between NSCLC and non-lung cancer.

\section{Materials and Methods}

\section{Study Population}

We continuously obtained 363 patients with NSCLC and 433 patients with non-lung cancer (healthy control group) from February 1, 2020 to June 30, 2021 in the Mindong Hospital of Fujian Medical University. The diagnosis of NSCLC mainly depends on the following: ${ }^{19}$ 1) age $>45$ years old with long-term smoking history and working or living history in a bad air environment; 2) Clinical symptoms: cough, hemoptysis, chest pain, chest tightness and discomfort, pulmonary inflammation, dyspnea, superior vena cava syndrome (upper limb edema or facial edema), extrapulmonary manifestations (joint pain and discomfort, syndrome of abnormal antidiuretic hormone secretion, etc.); 3) Imaging examination: conventional chest X-ray or CT examination found burr, lobulated, uneven density mass; 4) There were heterotypic cells in sputum; 5) Bronchoscopy: new organisms were found under a bronchoscope, and atypical cells were detected by brush or biopsy; 6) Under a bronchoscope, lymph node puncture was performed to find heterotypic cells; 7) PETCT: there was an abnormal metabolic area; 8) Patients who were difficult to identify clinically could have a mediastinoscopic or thoracoscopic biopsy to determine whether there were atypical cells. Among the above eight indexes, sputum examination, bronchoscopy, or lymph node puncture under bronchoscope are the gold indexes for the diagnosis of lung cancer. All included patients with NSCLC were divided into cases of squamous cell carcinoma $(\mathrm{N}=207)$, Adenocarcinoma $(\mathrm{N}=103)$, and large cell carcinoma $(\mathrm{N}=53)$. These patients with NSCLC consisted of stage I $(\mathrm{N}=34)$, stage II $(\mathrm{N}=143)$, stage IIIa $(\mathrm{N}=145)$, stage IIIb $(\mathrm{N}=24)$, and stage IV $(\mathrm{N}=17)$ For our purposes, patients with incomplete clinical data $(\mathrm{N}=83)$, chronic kidney disease or abnormal renal function $(\mathrm{N}=12)$, and a history of small cell lung cancer (SCLC) or other malignant tumor ( $\mathrm{N}=33$ ) were excluded. According to the guidelines of the Declaration of Helsinki, the Ethics Committee of Mindong Hospital of Fujian Medical University approved this study and all included patients have given informed consent.

\section{Laboratory Measurements}

Fasting venous blood was obtained after admission. Blood samples were measured for blood routine including the serum creatinine ( $\mathrm{SCr}$ ), hemoglobin $(\mathrm{Hb})$, and albumin (ALB), by using an automated blood counter (Sysmex XE5000; Emilio de Azevedo Campos, Porto, Portugal). The alanine transaminase (ALT) and aspartate transaminase (AST) by using the Siemens ADVIA 2400 automatic biochemistry analyzer (Siemens AG). Serum levels of HE4, CEA, SCCA, and CY21-1 were measured by ELISA Kit (Germany). The four serum biomarkers were measured three times and the average value was taken for data analysis. The CKD Epidemiology Collaboration 
(CKD-EPI) creatinine was used to calculate the estimated glomerular filtration rate (eGFR). ${ }^{20}$

\section{Statistical Analyses}

First, to evaluate associations between the four biomarkers (HE4, CEA, SCCA, and CY21-1) and risk of NSCLC, a corrected logical regression model was performed with four biomarkers as the independent variable and NSCLC as the dependent variable, respectively. Second, stratified analysis by adding "TNM stage" and "subtype of NSCLC" as covariates suggested significant associations between these four biomarkers and NSCLC. In these corrected logical regression models, Model 1 was adjusted for age and gender, Model 2 was adjusted for age, gender, and body mass index (BMI), Model 3 was adjusted for age, gender, BMI, and current smoker, and Model 4 was adjusted for age, gender, BMI, current smoker, current drinker, and biochemical detection ( $\mathrm{SCr}$, $\mathrm{Hb}, \mathrm{ALB}, \mathrm{ALT}$, and AST). Third, the predictive value of these biomarkers for risk of NSCLC was performed by receiver operating characteristic (ROC) curves and area under the curve (AUC). Sensitivity and specificity were calculated. Additionally, the combined prediction model, a 4-biomarker model (HE4, CEA, SCCA, and CY21-1), was constructed further to evaluate to risk of NSCLC. SPSS 26.0 and R 3.0 were used for the statistical analyses. $P \leq 0.05$ was defined as statistically significant.

\section{Result}

\section{Clinical Characteristics of All Included Patients on Admission}

The clinical characteristics of all included patients are presented (Table 1). Serum median values of HE4 (143.3 pmol/ L), CEA (4.60 ng/mL), SCCA (1.52 ng/mL), and CY21-1 $(5.36 \mathrm{ng} / \mathrm{mL})$ in patients with NSCLC were significantly higher than patients without NSCLC (all $P<0.05$ ). The patients with NSCLC were more likely to be current smokers, current drinkers, and had higher BMI and $\mathrm{SCr}$, and lower serum levels of $\mathrm{Hb}$ and ALB than patients without NSCLC (all $P<0.05$ ). There were no significant differences on serum ALT and AST levels between the two groups (all $P>0.05$ ).

\section{Elevated Serum Levels of these Biomarkers Had Significant Associations with Increased Risk of NSCLC}

To confirm the associations of these biomarkers (HE4, CEA, SCCA, and CY21-1) with risk of NSCLC on admission, corrected logistic regression models were used (Table 2). Multivariate logistic regression analysis revealed that increased serum levels of HE4 $(\mathrm{OR}=2.15,95 \% \mathrm{CI}=1.27$ 4.54, $P=0.012), \quad \mathrm{CEA} \quad(\mathrm{OR}=2.41,95 \% \quad \mathrm{CI}=1.53-5.57$, $P=0.001)$, SCCA ( $\mathrm{OR}=2.25,95 \% \mathrm{CI}=1.36-4.84, P=0.006)$, and $\mathrm{CY} 21-1(\mathrm{OR}=2.64,95 \% \mathrm{CI}=1.59-6.32, P<0.001)$ were significantly and independently associated after adjusting for age and gender in Model 1. When BMI, current smoker, current drinker, and biochemical detection $(\mathrm{SCr}, \mathrm{Hb}, \mathrm{ALB}$, ALT, and AST) were added into Model 1, our results showed that HE4 $(\mathrm{OR}=2.10,95 \% \mathrm{CI}=1.22-4.42, P=0.013)$, CEA $(\mathrm{OR}=2.30,95 \% \mathrm{CI}=1.44-4.53, P=0.004)$, SCCA $(\mathrm{OR}=2.20,95 \% \mathrm{CI}=1.29-4.55, P=0.011)$, and $\mathrm{CY} 21-1$ $(\mathrm{OR}=2.60,95 \% \mathrm{CI}=1.56-6.25, P<0.001)$ remained significantly and strongly associated with higher risk of NSCLC in Model 4.

\section{Stratified Analysis for Associations between the Four Biomarkers and Risk of NSCLC}

Stratified analysis, adding "TNM stage" and "subtype of NSCLC" as covariates, respectively, was performed to analyze association between the four biomarkers and risk of NSCLC. As shown in Table 3 (TNM stage as covariate), multivariate logistic regression analysis showed that serum HE4 (OR=2.10, 95\% $\mathrm{CI}=1.22-4.45, P=0.013)$, CEA (OR=2.36, 95\% CI=1.45-4.67, $P=0.004)$, SCCA $(\mathrm{OR}=2.20,95 \% \mathrm{CI}=1.29-4.55, P=0.011)$, and $\mathrm{CY} 21-1$ $(\mathrm{OR}=2.61,95 \% \mathrm{CI}=1.55-6.24, P<0.001)$ were still significantly associated with elevated risk of NSCLC after adjusting for age, gender, BMI, current smoker, current drinker, and biochemical detection ( $\mathrm{SCr}, \mathrm{Hb}, \mathrm{ALB}, \mathrm{ALT}$, and AST) in Model 4. Similarly, as shown in Table 4 (subtype of NSCLC as covariate), our study suggested that serum HE4 $(\mathrm{OR}=2.11,95 \% \mathrm{CI}=1.27-4.28, P=0.013)$, CEA $(\mathrm{OR}=2.31,95 \% \mathrm{CI}=1.45-4.56, P=0.005)$, SCCA $(\mathrm{OR}=2.21,95 \% \mathrm{CI}=1.29-4.58, P=0.011)$, and $\mathrm{CY} 21-1$ $(\mathrm{OR}=2.62,95 \% \mathrm{CI}=1.55-6.28, P<0.001)$ were still significantly associated with elevated risk of NSCLC after adjusting for age, gender, BMI, current smoker, current drinker, and biochemical detection ( $\mathrm{SCr}, \mathrm{Hb}, \mathrm{ALB}, \mathrm{ALT}$, and AST) in Model 4.

\section{The Four Biomarkers Had Predictive Value for Detecting NSCLC}

To determine the predictive value of biomarkers (HE4, CEA, SCCA, and CY21-1) for NSCLC, ROC-AUC 
Table I Admission Characteristics of Patients with NSCLC

\begin{tabular}{|c|c|c|c|}
\hline Variables & With NSCLC (N=363) & Healthy Control Group $(\mathrm{N}=433)$ & $P$-value \\
\hline Age (years) & $66.0(60.0-72.0)$ & $64.8(57.3-70.4)$ & 0.061 \\
\hline Gender (male), n (\%) & $260(71.6)$ & $288(66.5)$ & 0.109 \\
\hline BMI $\left(\mathrm{kg} / \mathrm{m}^{2}\right)$ & $22.4 \pm 3.5$ & $25.5 \pm 7.2$ & 0.004 \\
\hline Current smoker, n (\%) & $255(70.2)$ & $136(31.4)$ & $<0.001$ \\
\hline Current drinker, n (\%) & $133(36.6)$ & $176(40.6)$ & 0.036 \\
\hline \multicolumn{4}{|l|}{ TNM stage } \\
\hline Stage I, n (\%) & $34(9.4)$ & - & \\
\hline Stage II, n (\%) & $143(39.4)$ & _ & \\
\hline Stage IIIA, n (\%) & $145(39.9)$ & - & \\
\hline Stage IIIB, n (\%) & $24(6.6)$ & - & \\
\hline Stage IV, n (\%) & $17(4.7)$ & - & \\
\hline Subtype of NSCLC & & - & \\
\hline Squamous cell carcinoma, n (\%) & $207(57.0)$ & - & \\
\hline Adenocarcinoma, n (\%) & $103(28.4)$ & - & \\
\hline Large cell carcinoma, n (\%) & $53(14.6)$ & - & \\
\hline \multicolumn{4}{|l|}{ Biochemical detection } \\
\hline CEA $(n g / m L)$ & $4.60(2.30-18.0)$ & $3.11(1.33-14.2)$ & 0.004 \\
\hline $\operatorname{SCCA}(\mathrm{ng} / \mathrm{mL})$ & $1.52(0.99-3.03)$ & $1.13(0.77-2.84)$ & $<0.001$ \\
\hline CY2I-I (ng/mL) & $5.36(3.09-10.43)$ & $4.17(2.52-9.62)$ & $<0.001$ \\
\hline HE4 (pmol/L) & |43.3 (| | 3.5-|784.I) & $70.6(42.6-87.9)$ & $<0.001$ \\
\hline $\mathrm{SCr}(\mu \mathrm{mol} / \mathrm{L})$ & $88.9(75.3-93.6)$ & $85.4(73.5-91.2)$ & 0.014 \\
\hline $\mathrm{Hb}(\mathrm{g} / \mathrm{L})$ & $112(105-128)$ & $123(113-135)$ & $<0.001$ \\
\hline ALB $(g / L)$ & $35.8(34.1-40.5)$ & $45.4(39.2-51.7)$ & $<0.001$ \\
\hline ALT (U/L) & $17(11-24)$ & $18(13-27)$ & 0.314 \\
\hline AST (U/L) & $21(16-24)$ & $23(18-28)$ & 0.191 \\
\hline
\end{tabular}

Notes: Normally distributed data are presented as the mean $\pm S D$, non-normally distributed data are presented as the median (interquartile range), and categorical variables are presented as the $n(\%)$.

Abbreviations: NSCLC, non-small cell lung cancer; BMI, body mass index; HE4, human epididymis protein 4; CEA, carcinoembryonic antigen; SCCA, squamous cell carcinoma antigen; CY2I-I, cytokeratin 19 fragment; $\mathrm{Hb}$, hemoglobin; $\mathrm{SCr}$, serum creatinine; ALB, albumin; ALT alanine transaminase; AST, aspartate transaminase.

Table 2 The Association between Serum Biomarkers and NSCLC by Logistic Regression Analysis

\begin{tabular}{|c|c|c|c|c|}
\hline Variables & Model I & Model 2 & Model 3 & Model 4 \\
\hline CEA (ng/mL); Increased per SD & $2.4 \mathrm{I}(1.53-5.57)$ & $2.36(1.48-5.40)$ & $2.32(1.45-5.34)$ & $2.30(1.44-4.53)$ \\
\hline$P$-value & 0.001 & 0.003 & 0.004 & 0.004 \\
\hline SCCA (ng/mL); Increased per SD & $2.25(1.36-4.84)$ & $2.23(1.34-4.62)$ & $2.21(1.30-4.59)$ & $2.20(1.29-4.55)$ \\
\hline$P$-value & 0.006 & 0.008 & 0.009 & 0.011 \\
\hline CY2I-I (ng/mL); Increased per SD & $2.64(1.59-6.32)$ & $2.62(1.58-6.30)$ & $2.61(1.57-6.29)$ & $2.60(1.56-6.25)$ \\
\hline$P$-value & $<0.001$ & $<0.001$ & $<0.001$ & $<0.001$ \\
\hline HE4 (pmol/L); Increased per SD & $2.15(1.27-4.54)$ & $2.14(1.26-4.54)$ & $2.12(1.25-4.46)$ & $2.10(1.22-4.42)$ \\
\hline$P$-value & 0.012 & 0.012 & 0.013 & 0.013 \\
\hline
\end{tabular}

Notes: Model I: Adjusted for age and gender. Model 2: Adjusted for age, gender, and BMI. Model 3: Adjusted for age, gender, BMI, and current smoker. Model 4: Adjusted for age, gender, BMI, current smoker and current drinker, and biochemical detection $(\mathrm{SCr}, \mathrm{Hb}, \mathrm{ALB}, \mathrm{ALT}$, and AST).

Abbreviations: NSCLC, non-small cell lung cancer; BMl, body mass index; HE4, human epididymis protein 4; CEA, carcinoembryonic antigen; SCCA, squamous cell carcinoma antigen; CY2I-I, cytokeratin 19 fragment; $\mathrm{Hb}$, hemoglobin; SCr, serum creatinine; ALB, albumin; ALT alanine transaminase; AST, aspartate transaminase. 
Table 3 The Association between Serum Biomarkers and NSCLC Stratified by TNM Stage

\begin{tabular}{|c|c|c|c|c|c|}
\hline Variables & Stage I & Stage II & Stage IIIA & Stage IIIB & Stage IV \\
\hline CEA (increased per SD) & $2.38(I .46-4.7 I)$ & $2.33(1.43-4.69)$ & $2.29(1.34-4.53)$ & $2.32(1.37-4.62)$ & $2.36(1.45-4.67)$ \\
\hline$P$-value & 0.004 & 0.005 & 0.06 & 0.005 & 0.004 \\
\hline SCCA (increased per SD) & $2.21(1.29-4.56)$ & $2.18(1.27-4.53)$ & $2.19(1.29-4.53)$ & $2.22(1.30-4.63)$ & $2.20(1.29-4.55)$ \\
\hline$P$-value & 0.010 & 0.014 & 0.011 & 0.010 & 0.011 \\
\hline CY2I-I (increased per SD) & $2.63(I .56-6.4 I)$ & $2.64(1.57-6.59)$ & $2.57(1.53-6.10)$ & $2.58(1.52-6.01)$ & $2.6 \mathrm{I}(1.55-6.24)$ \\
\hline$P$-value & $<0.001$ & $<0.001$ & $<0.001$ & $<0.001$ & $<0.001$ \\
\hline HE4 (increased per SD) & $2.12(1.23-4.53)$ & $2.11(1.22-4.46)$ & $2.08(1.21-4.37)$ & $2.09(1.2 \mid-4.53)$ & $2.10(1.22-4.45)$ \\
\hline$P$-value & 0.012 & 0.012 & 0.014 & 0.013 & 0.013 \\
\hline
\end{tabular}

Notes: Adjusted for age, gender, BMI, current smoker and current drinker, and biochemical detection (SCr, Hb, ALB, ALT, and AST).

Abbreviations: NSCLC, non-small cell lung cancer; BMI, body mass index; HE4, human epididymis protein 4; CEA, carcinoembryonic antigen; SCCA, squamous cell carcinoma antigen; CY2I-I, cytokeratin 19 fragment; Hb, hemoglobin; SCr, serum creatinine; ALB, albumin; ALT alanine transaminase; AST, aspartate transaminase.

analysis was performed (Figure 1). The HE4 (AUC $=0.862$, 95\% $\mathrm{CI}=0.795-0.929, P<0.001)$ had a better predictive value than $\mathrm{CY} 21-1 \quad(\mathrm{AUC}=0.778,95 \% \mathrm{CI}=0.694-0.863$, $P<0.001$ ), SCCA (AUC $=0.647,95 \% \quad \mathrm{CI}=0.540-0.754$, $P=0.008$ ), and CEA (AUC $=0.559,95 \% \mathrm{CI}=0.454-0.665$, $P=0.045$ ) on predicting NSCLC (Table 5). Additionally, the predictive model (CEA+SCCA+CY21-1+HE4) had an ideal capability to detecting existence of NSCLC (AUC $=0.954,95 \% \mathrm{CI}=0.927-0.999, P<0.001)$ in Table 6 .

\section{Discussion}

This study demonstrated that high HE4 levels independently contributed to higher risk of NSCLC in a Chinese elderly population. Well-known clinical features, such as age, tumor stage, pathological classification of NSCLC, smoking, and drinking history, did not affect the independent association, as well as CEA, SCCA, and CY21-1. ROC-AUC analysis showed that serum HE4 had good predictive value for detecting NSCLC, like in ovarian cancer. $^{21}$ Our results further suggested that the predictive model (CEA+SCCA +CY21-1+HE4) had an ideal capability to detectNSCLC.

Previous studies have confirmed that serum HE4 can be considered as a biomarker for the formation and recurrence of ovarian cancer in female patients. ${ }^{21}$ Importantly, as a multitissue- expressed protein, HE4 was also found to be involved in immune regulation and inflammatory response in

Table 4 The Association between Serum Biomarkers and NSCLC Stratified by Subtype of NSCLC

\begin{tabular}{|c|c|c|c|}
\hline Variables & Squamous Cell Carcinoma & Adenocarcinoma & Large Cell Carcinoma \\
\hline CEA (ng/mL); Increased per SD & $2.35(1.47-5.63)$ & $2.24(1.32-4.43)$ & $2.31(1.45-4.56)$ \\
\hline$P$-value & $<0.001$ & 0.010 & 0.005 \\
\hline SCCA (ng/mL); Increased per SD & $2.27(1.34-4.98)$ & $2.10(1.22-4.19)$ & $2.21(1.29-4.58)$ \\
\hline$P$-value & 0.002 & 0.011 & 0.011 \\
\hline CY2I-I (ng/mL); Increased per SD & $2.68(1.64-6.78)$ & $2.46(I .50-6.01)$ & $2.62(1.55-6.28)$ \\
\hline$P$-value & $<0.001$ & $<0.001$ & $<0.001$ \\
\hline HE4 (pmol/L); Increased per SD & $2.16(I .24-4.7 I)$ & $2.02(1.19-4.25)$ & $2.11(1.27-4.28)$ \\
\hline$P$-value & 0.012 & 0.017 & 0.013 \\
\hline
\end{tabular}

Notes: Adjusted for age, gender, BMI, current smoker and current drinker, and biochemical detection (SCr, Hb, ALB, ALT, and AST).

Abbreviations: NSCLC, non-small cell lung cancer; BMI, body mass index; HE4, human epididymis protein 4; CEA, carcinoembryonic antigen; SCCA, squamous cell carcinoma antigen; CY2I-I, cytokeratin 19 fragment; Hb, hemoglobin; SCr, serum creatinine; ALB, albumin; ALT alanine transaminase; AST, aspartate transaminase. 


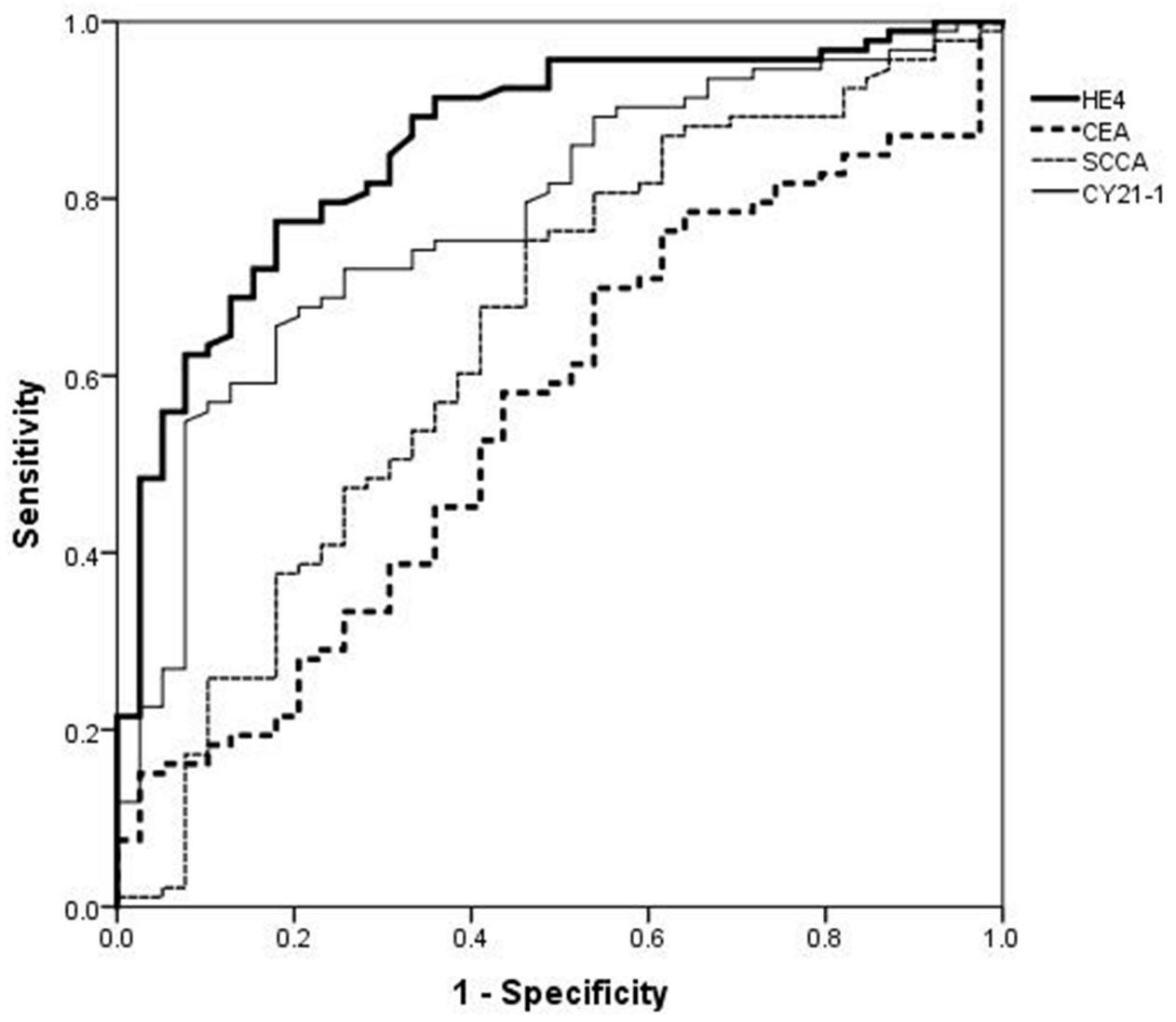

Figure I Predictive value $f$ of four serum biomarkers on detecting NSCLC by ROC curve.

the upper respiratory tract and lung. Existing investigations have suggested that in patients with NSCLC, the expression of HE4 in the cancer tissue is elevated and the serum level of HE4 is increased. ${ }^{22-24}$ One study further found that HE4 expression in NSCLC tissue was significantly higher than lung benign diseases in female patients. ${ }^{14}$ The ROC curve in their study showed that serum HE4 presented the AUC value of 0.78 with a high sensitivity of $91 \%$ and a low specificity of $61 \% .{ }^{14}$ However, another study also reported that serum HE4 has the AUC value of 0.988 on predicting NSCLC. ${ }^{24}$ These

Table 5 Predictive Value for Detecting NSCLC

\begin{tabular}{|l|c|c|c|c|}
\hline Variables & AUC & $\begin{array}{c}\text { Standard } \\
\text { Error }\end{array}$ & 95\% Cl & P-value \\
\hline CEA $(\mathrm{ng} / \mathrm{mL})$ & 0.559 & 0.054 & $0.454-0.665$ & 0.045 \\
SCCA $(\mathrm{ng} / \mathrm{mL})$ & 0.647 & 0.055 & $0.540-0.754$ & 0.008 \\
CY2I-I (ng/mL) & 0.778 & 0.043 & $0.694-0.863$ & $<0.001$ \\
HE4 $(\mathrm{pmol} / \mathrm{L})$ & 0.862 & 0.034 & $0.795-0.929$ & $<0.00 \mathrm{I}$ \\
\hline
\end{tabular}

Abbreviations: NSCLC, non-small cell lung cancer; HE4, human epididymis protein 4; CEA, carcinoembryonic antigen; SCCA, squamous cell carcinoma antigen; CY2I-I, cytokeratin 19 fragment; AUC, area under the curve. previous findings are not consistent with our results that only have the AUC of 0.862 . This discrepancy is mainly due to different selection methods of populations, different criteria of inclusion and exclusion, and analysis methods between our study and previous studies. Importantly, our predictive model (CEA+SCCA+CY21-1+HE4) had a good capability to detecting the existence of NSCLC (AUC $=0.954,95 \%$ $\mathrm{CI}=0.927-0.999, \quad P<0.001)$. The combined diagnostic model of these markers further improves the diagnostic value of HE4 on detecting NSCLC.

Table 6 Predictive Models for Detecting NSCLC

\begin{tabular}{|l|c|c|c|c|}
\hline Variables & AUC & $\begin{array}{c}\text { Standard } \\
\text { Error }\end{array}$ & $\mathbf{9 5 \%} \mathbf{~ C l}$ & P-value \\
\hline CEA+HE4 & 0.872 & $0.03 \mathrm{I}$ & $0.799-0.944$ & $<0.00 \mathrm{I}$ \\
SCCA+HE4 & 0.889 & 0.030 & $0.803-0.959$ & $<0.001$ \\
CY2I-I+HE4 & 0.894 & 0.027 & $0.805-0.974$ & $<0.001$ \\
CEA+SCCA & 0.954 & $0.02 \mathrm{I}$ & $0.927-0.999$ & $<0.00 \mathrm{I}$ \\
+CY2I-I+HE4 & & & & \\
\hline
\end{tabular}

Abbreviations: NSCLC, non-small cell lung cancer; HE4, human epididymis protein 4; CEA, carcinoembryonic antigen; SCCA, squamous cell carcinoma antigen; CY2I-I, cytokeratin 19 fragment; AUC, area under the curve. 
Additionally, previous studies have also demonstrated that expression of HE4 protein is involved in renal fibrosis and can be used as a sensitive indicator of abnormal renal function. ${ }^{25-28}$ Thus, in order to eliminate the interference of renal function, patients with CKD or renal abnormalities were excluded in our study. In the multivariate analysis models, we found that there was still an independent association between HE4 and NSCLC after correcting SCr. The elevated HE4 levels have also been found to be related to abnormal cardiac function, but there is no direct evidence to prove that the increased expression of HE4 in cardiac tissue was involved in cardiac insufficiency. ${ }^{29-31}$ These studies speculate that the acute renal injury caused by cardiac insufficiency further leads to the increase of serum HE4.

This study has several important advantages. On the one hand, because HE4 is related to a variety of diseases, such as cancers (ovarian and cervical cancer), abnormal renal function (chronic kidney disease, acute kidney injury), acute or chronic heart failure, fibrosis, and inflammation, ${ }^{14,22-31}$ we firstly used a combined prediction model $(\mathrm{CEA}+\mathrm{SCCA}+\mathrm{CY} 21-1+\mathrm{HE} 4)$ to evaluate its predictive value on detecting NSCLC. The combined prediction model significantly improved the predictive value of HE4 on detecting NSCLC by increasing the specificity. On the other hand, these serum biomarkers (CEA+SCCA+CY21-1+HE4) were detected for three times in each patient within 24 hours of admission. The average value of the three times is included in our analysis to ensure the reliability and stability of the data. Of course, this study has some limitations. First, this is a cross-sectional analysis with a small sample and did not confirm the causal link between the predictive model of HE4 and NSCLC. Second, patients with chronic kidney disease or abnormal renal function and history of SCLC or other malignant tumor were excluded in our study. Our prediction model is not applicable to patients with these diseases, which limits the implementation of the prediction model.

\section{Conclusion}

In summary, our findings provided the first evidence that serum HE4 levels have a significant association with risk of NSCLC in a Chinese elderly population. The predictive model (CEA+SCCA+CY21-1+HE4) had an ideal capability to predict NSCLC.

\section{Funding}

This study was supported by the Startup Fund for Scientific Research, Fujian Medical University(Grant number:2019QH1219).

\section{Disclosure}

The authors report no conflicts of interest in this work.

\section{References}

1. Xue C, Hu Z, Jiang W, et al. National survey of the medical treatment status for non-small cell lung cancer (NSCLC) in China. Lung Cancer. 2012;77(2):371-375. doi:10.1016/j.lungcan.2012.04.014

2. Torre LA, Siegel RL, Ward EM, Jemal A. Global cancer incidence and mortality rates and trends-an update. Cancer Epidemiol Biomarkers Prev. 2016;25(1):16-27. doi:10.1158/1055-9965.EPI-15-0578

3. Kulpa J, Wójcik E, Reinfuss M, Kołodziejski L. Carcinoembryonic antigen, squamous cell carcinoma antigen, CYFRA 21-1, and neuron-specific enolase in squamous cell lung cancer patients. Clin Chem. 2002;48(11):1931-1937. doi:10.1093/clinchem/48.11.1931

4. Diamandis EP, Hoffman BR, Sturgeon CM. National Academy of Clinical Biochemistry Laboratory Medicine practice guidelines for the use of tumor markers. Clin Chem. 2008;54(11):1935-1939. doi:10.1373/clinchem.2008.105494

5. Wang X, Fan Y, Wang J, Wang H, Liu W. Evaluating the expression and diagnostic value of human epididymis protein 4 (HE4) in small cell lung cancer. Tumour Biol. 2014;35(7):6847-6853. doi:10.1007/ s13277-014-1943-8

6. Speeckaert MM, Speeckaert R, Delanghe JR. Human epididymis protein 4 in cancer diagnostics: a promising and reliable tumor marker. Adv Clin Chem. 2013;59:1-21.

7. Scaletta G, Plotti F, Luvero D, et al. The role of novel biomarker HE4 in the diagnosis, prognosis and follow-up of ovarian cancer: a systematic review. Expert Rev Anticancer Ther. 2017;17 (9):827-839. doi:10.1080/14737140.2017.1360138

8. Lu KH, Patterson AP, Wang L, et al. Selection of potential markers for epithelial ovarian cancer with gene expression arrays and recursive descent partition analysis. Clin Cancer Res. 2004;10 (10):3291-3300. doi:10.1158/1078-0432.CCR-03-0409

9. Lawton AJ, Lee KA, Cheville AL, et al. Assessment and management of patients with metastatic spinal cord compression: a multidisciplinary review. J Clin Oncol. 2019;37(1):61-71. doi:10.1200/JCO.2018.78.1211

10. Karlsen NS, Karlsen MA, Høgdall CK, Høgdall EV. HE4 tissue expression and serum HE4 levels in healthy individuals and patients with benign or malignant tumors: a systematic review. Cancer Epidemiol Biomarkers Prev. 2014;23(11):2285-2295. doi:10.1158/ 1055-9965.EPI-14-0447

11. Kristjansdottir B, Levan K, Partheen K, Sundfeldt K. Diagnostic performance of the biomarkers HE4 and CA125 in type I and type II epithelial ovarian cancer. Gynecol Oncol. 2013;131(1):52-58. doi:10.1016/j.ygyno.2013.07.094

12. Kamei M, Yamashita S, Tokuishi K, et al. HE4 expression can be associated with lymph node metastases and disease-free survival in breast cancer. Anticancer Res. 2010;30(11):4779-4783.

13. Lan WG, Hao YZ, Xu DH, Wang P, Zhou YL, Ma LB. Serum human epididymis protein 4 is associated with the treatment response of concurrent chemoradiotherapy and prognosis in patients with locally advanced non-small cell lung cancer. Clin Transl Oncol. 2016;18 (4):375-380. doi:10.1007/s12094-015-1375-y

14. Lamy PJ, Plassot C, Pujol JL, de Mello RA. Serum HE4: an independent prognostic factor in non-small cell lung cancer. PLoS One. 2015;10(6):e0128836. doi:10.1371/journal.pone.0128836 
15. Jiang Y, Wang C, Lv B, Ma G, Wang L. Expression level of serum human epididymis 4 and its prognostic significance in human non-small cell lung cancer. Int J Clin Exp Med. 2014;7(12):5568-5572.

16. Liu W, Yang J, Chi PD, et al. Evaluating the clinical significance of serum HE4 levels in lung cancer and pulmonary tuberculosis. Int J Tuberc Lung Dis. 2013;17(10):1346-1353. doi:10.5588/ ijtld.13.0058

17. Yamashita S, Tokuishi K, Hashimoto T, et al. Prognostic significance of HE4 expression in pulmonary adenocarcinoma. Tumour Biol. 2011;32(2):265-271. doi:10.1007/s13277-010-0118-5

18. Lou E, Johnson M, Sima C, et al. Serum biomarkers for assessing histology and outcomes in patients with metastatic lung cancer. Cancer Biomark. 2014;14(4):207-214. doi:10.3233/CBM-140399

19. Duma N, Santana-Davila R, Molina JR. Non-small cell lung cancer: epidemiology, screening, diagnosis, and treatment. Mayo Clin Proc. 2019;94(8):1623-1640. doi:10.1016/j.mayocp.2019.01.013

20. Stevens PE. Evaluation and management of chronic kidney disease: synopsis of the kidney disease: improving global outcomes 2012 clinical practice guideline. Ann Intern Med. 2013;158(11):825. doi:10.7326/0003-4819-158-11-201306040-00007

21. Potenza E, Parpinel G, Laudani ME, Macchi C, Fuso L, Zola P. Prognostic and predictive value of combined HE-4 and CA-125 biomarkers during chemotherapy in patients with epithelial ovarian cancer. Int J Biol Markers. 2020;35(4):20-27. doi:10.1177/ 1724600820955195

22. Escudero JM, Auge JM, Filella X, Torne A, Pahisa J, Molina R. Comparison of serum human epididymis protein 4 with cancer antigen 125 as a tumor marker in patients with malignant and nonmalignant diseases. Clin Chem. 2011;57(11):1534-1544. doi:10.1373/ clinchem.2010.157073
23. Mo D, He F. Serum human epididymis secretory protein 4 (HE4) is a potential prognostic biomarker in non-small cell lung cancer. Clin Lab. 2018;64(4):1421-1428. doi:10.7754/Clin.Lab.2018.180222

24. Li LM, Zhu YX, Zhong Y, et al. Human epididymis protein 4 in endometrial cancer: a meta-analysis. Clin Chim Acta. 2018;482:215-223. doi:10.1016/j.cca.2018.03.040

25. LeBleu VS, Teng Y, O'Connell JT, et al. Identification of human epididymis protein-4 as a fibroblast-derived mediator of fibrosis. Nat Med. 2013;19(2):227-231. doi:10.1038/nm.2989

26. Luo J, Wang F, Wan J, et al. Serum human epididymis secretory protein 4 as a potential biomarker of renal fibrosis in kidney transplantation recipients. Clin Chim Acta. 2018;483:216-221. doi:10.1016/j.cca.2018.05.006

27. Yuan T, Li Y. Human epididymis protein 4 as a potential biomarker of chronic kidney disease in female patients with normal ovarian function. Lab Med. 2017;48(3):238-243. doi:10.1093/labmed/lmx036

28. Tajima S, Fu R, Shigematsu T, et al. Urinary human epididymis secretory protein 4 as a useful biomarker for subclinical acute rejection three months after kidney transplantation. Int J Mol Sci. 2019;20 (19):4699. doi:10.3390/ijms20194699

29. Piek A, Meijers WC, Schroten NF, Gansevoort RT, de Boer RA, Silljé HH. HE4 serum levels are associated with heart failure severity in patients with chronic heart failure. J Card Fail. 2017;23(1):12-19. doi:10.1016/j.cardfail.2016.05.002

30. Huang Y, Jiang H, Zhu L. Human epididymis protein 4 as an indicator of acute heart failure in patients with chronic kidney disease. Lab Med. 2020;51(2):169-175.

31. de Boer RA, Cao Q, Postmus D, et al. The WAP four-disulfide core domain protein HE4: a novel biomarker for heart failure. JACC Heart Fail. 2013;1(2):164-169. doi:10.1016/j.jchf.2012.11.005
International Journal of General Medicine

\section{Publish your work in this journal}

The International Journal of General Medicine is an international, peer-reviewed open-access journal that focuses on general and internal medicine, pathogenesis, epidemiology, diagnosis, monitoring and treatment protocols. The journal is characterized by the rapid reporting of reviews, original research and clinical studies

\section{Dovepress}

across all disease areas. The manuscript management system is completely online and includes a very quick and fair peer-review system, which is all easy to use. Visit http://www.dovepress.com/ testimonials.php to read real quotes from published authors. 